\title{
The Exact and Asymptotic Distributions of Cramér-von Mises Statistics
}

\author{
By SÁNDOR CSÖRGŐ $†$ and JULIAN J. FARAWAY \\ University of Michigan, Ann Arbor, USA
}

[Received February 1994. Revised December 1994]

\begin{abstract}
SUMMARY
It is shown that an asymptotically precise one-term correction to the asymptotic distribution function of the classical Cramér-von Mises statistic approximates the exact distribution function remarkably closely for sample sizes as small as 7 or even smaller. This correction can be quickly evaluated, and hence it is suitable for the computation of practically exact $p$-values when testing simple goodness of fit. Similar findings hold for Watson's rotationally invariant modification, where a sample size of 4 appears to suffice.
\end{abstract}

Keywords: ASYMPTOTIC EXPANSIONS; CRAMÉR-VON MISES STATISTIC; SMALL SAMPLE DISTRIBUTIONS; WATSON'S STATISTIC

\section{ASYMPTOTIC THEORY FOR $\omega_{n}^{2}$}

Let $X_{1}, \ldots, X_{n}$ be independent identically distributed random variables with pertaining sample distribution function $F_{n}(x)=n^{-1} \#\left\{1 \leqslant k \leqslant n: X_{k} \leqslant x\right\}, x \in \mathbf{R}$. The Cramér-von Mises statistic for testing whether the random variables have distribution function $F()$, assumed to be a continuous function on the real line $\mathbf{R}$, is

$$
\omega_{n}^{2}=n \int_{-\infty}^{\infty}\left\{F_{n}(x)-F(x)\right\}^{2} \mathrm{~d} F(x)=n \int_{0}^{1}\left\{G_{n}(s)-s\right\}^{2} \mathrm{~d} s,
$$

where $G_{n}(s)=n^{-1} \#\left\{1 \leqslant k \leqslant n: U_{k} \leqslant s\right\}, 0 \leqslant s \leqslant 1$, is the empirical distribution function of the independent random variables $U_{1}=F\left(X_{1}\right), \ldots, U_{n}=F\left(X_{n}\right)$, uniformly distributed in the interval $[0,1]$. If $U_{1, n} \leqslant \ldots \leqslant U_{n, n}$ denote the corresponding uniform $[0,1]$ order statistics, then the latter form can be integrated out to obtain

$$
\frac{1}{12 n} \leqslant \omega_{n}^{2}=\frac{1}{12 n}+\sum_{k=1}^{n}\left(U_{k, n}-\frac{2 k-1}{2 n}\right)^{2} \leqslant \frac{n}{3}, \quad n=1,2, \ldots,
$$

where the bounds are achieved with probability 0 , the upper bound $n / 3$ when either $U_{n, n}=0$ or $U_{1, n}=1$. Hence for the distribution functions $V_{n}(x)=P\left\{\omega_{n}^{2} \leqslant x\right\}, x \in \mathbf{R}$, we have $V_{n}(x)=0$ if $x \leqslant 1 / 12 n$ and $V_{n}(x)=1$ if $x \geqslant n / 3$.

Statistics similar to $\omega_{n}^{2}$ were proposed by Cramér (1928), pages 145-147, and von Mises (1931), pages 316-335. The distribution-free variant $\omega_{n}^{2}$ itself was introduced by Smirnov (1936, 1937), giving credit to V. I. Glivenko in a footnote on p.974 in the 1937 paper. With $\mathrm{i}$ denoting the imaginary unit, Smirnov proved that, for all $t \in \mathbf{R}$,

$\dagger$ Address for correspondence: Department of Statistics, 1444 Mason Hall, University of Michigan, 419 South State Street, Ann Arbor, MI 48109-1027, USA.

E-mail: scsorgo@stat.lsa.umich.edu 


$$
\begin{aligned}
\lim _{n \rightarrow \infty}\left[E\left\{\exp \left(\mathrm{i} t \omega_{n}^{2}\right)\right\}\right] & =\lim _{n \rightarrow \infty}\left\{\int_{1 / 12 n}^{n / 3} \exp (\mathrm{i} t x) \mathrm{d} V_{n}(x)\right\}=\left\{\frac{(-2 \mathrm{i} t)^{1 / 2}}{\sinh (-2 \mathrm{i} t)^{1 / 2}}\right\}^{1 / 2}=\varphi(t) \\
& =\int_{0}^{\infty} \exp (\mathrm{i} t x) \mathrm{d} V(x)
\end{aligned}
$$

A related result is by von Mises (1947) for his version of $\omega_{n}^{2}$. Smirnov's result in equation (1.2) implies that $\lim _{n \rightarrow \infty}\left\{V_{n}(x)\right\}=V(x), x \in \mathbf{R}$, where $V(x)=0$ if $x \leqslant 0$, and $\varphi()$ was also inverted by Smirnov $(1936,1937)$, obtaining the first formula

$$
\begin{aligned}
V(x) & =1-\frac{2}{\pi} \sum_{k=1}^{\infty}(-1)^{k+1} \int_{(2 k-1) \pi}^{2 k \pi} \frac{\exp \left(-u^{2} x / 2\right)}{(-u \sin u)^{1 / 2}} \mathrm{~d} u \\
& =\frac{1}{\pi^{3 / 2} x^{1 / 2}} \sum_{k=0}^{\infty} \frac{\Gamma\left(k+\frac{1}{2}\right)}{k !}(4 k+1)^{1 / 2} \exp \left\{-\frac{(4 k+1)^{2}}{16 x}\right\} K_{1 / 4}\left\{\frac{(4 k+1)^{2}}{16 x}\right\} \\
& =\frac{2}{\pi^{1 / 2} x^{1 / 4}} \sum_{k=0}^{\infty}(-1)^{k}\left(\begin{array}{c}
-\frac{1}{2} \\
k
\end{array}\right) \exp \left\{-\frac{(4 k+1)^{2}}{16 x}\right\} D_{-1 / 2}\left(\frac{4 k+1}{2 x^{1 / 2}}\right), \quad x>0,
\end{aligned}
$$

where $\Gamma(y)=\int_{0}^{\infty} u^{y-1} \exp (-u) \mathrm{d} u, y>0$, is the usual gamma function and $K_{\nu}()$, $\nu>-\frac{1}{2}$, is a modified Bessel function of the third kind (Watson (1966), p. 172):

$$
K_{\nu}(y)=\frac{\pi^{1 / 2} y^{\nu}}{2^{\nu} \Gamma\left(\nu+\frac{1}{2}\right)} \int_{0}^{\infty} \exp (-y \cosh u) \sinh ^{2 \nu}(u) \mathrm{d} u, \quad y>0,
$$

and $D_{v}(u), u \in \mathbf{R}$, is the parabolic cylinder function of index $v \in \mathbf{R}$ (Abramowitz and Stegun (1973), p. 687). The second formula is due to Anderson and Darling (1952), who also identified $V()$ as the distribution function of the random variable $\omega^{2}=$ $\int_{0}^{1} B^{2}(s) \mathrm{d} s$, where $\{B(s): 0 \leqslant s \leqslant 1\}$ is a Brownian bridge, and compiled the first table of quantiles of $V$. The third line in equation (1.3) results from the second by the case $l=1$ of the identity

$$
(-1)^{k}\left(\begin{array}{c}
-l / 2 \\
k
\end{array}\right)=\frac{\Gamma(k+l / 2)}{k ! \Gamma(l / 2)}, \quad k=0,1,2, \ldots, \quad l=1,2, \ldots,
$$

and that $\Gamma\left(\frac{1}{2}\right)=\pi^{1 / 2}$, and by the first of the identities $D_{-1 / 2}\left(2 u^{1 / 2}\right)=u^{1 / 4} K_{1 / 4}(u) / \pi^{1 / 2}$,

$$
\begin{gathered}
D_{1 / 2}\left(2 u^{1 / 2}\right)=\frac{u^{3 / 4}}{\pi^{1 / 2}}\left\{K_{1 / 4}(u)+K_{3 / 4}(u)\right\}, \\
D_{3 / 2}\left(2 u^{1 / 2}\right)=\frac{u^{5 / 4}}{\pi^{1 / 2}}\left\{2 K_{1 / 4}(u)+3 K_{3 / 4}(u)-K_{5 / 4}(u)\right\}
\end{gathered}
$$

for all $u>0$ (Abramowitz and Stegun (1973), p. 692). The most extensive table for the values of $V()$ to date has been computed by Martynov (1978), pages 63-64. 
For possible refinements of the limit theorem $\Delta_{n}=\sup \left\{\left|V_{n}(x)-V(x)\right|: x \in \mathbf{R}\right\}$ $\rightarrow 0$, Darling (1960) proposed, without proof, the expansion $E\left\{\exp \left(-z \omega_{n}^{2}\right)\right\}$ $\approx E\left\{\exp \left(-z \omega^{2}\right)\right\}+a(z) n^{-1}, z \geqslant 0$, where extending $\varphi()$ in equation (1.2) for complex arguments and setting $\xi(z)=\varphi(\mathrm{iz})$,

$$
a(z)=\frac{1}{12}\left[\xi(z)-\left\{\frac{1}{3}+\frac{7}{24} \cosh (2 z)^{1 / 2}\right\} \xi^{3}(z)-\left\{\frac{1}{3}+\frac{1}{24} \cosh (8 z)^{1 / 2}\right\} \xi^{5}(z)\right] .
$$

Then, in the period 1965-74, improving results for the rate of convergence were obtained in the form $\Delta_{n}=O\left(r_{n}\right)$, with $r_{n}=(\log n)^{-1 / 4}$ by Kandelaki, $r_{n}=n^{\epsilon-1 / 10}$ and $r_{n}=n^{\epsilon-1 / 6}$ in two papers by Sazonov, $r_{n}=(\log n)^{3 / 2} n^{-1 / 5}$ by Rosenkrantz, $r_{n}=$ $(\log n)^{3 / 2} n^{-1 / 4}$ by Kiefer, $r_{n}=(\log n)^{5 / 4} n^{-1 / 4}$ by Nikitin, $r_{n}=n^{\epsilon-1 / 3}$ by Orlov, for any $\epsilon>0$, and finally $r_{n}=(\log n) n^{-1 / 2}$ in Csörgo (1976) that contains the seven references. The last $r_{n}$ is still far from accurate. Csörgö $(1975,1976)$ also established an expansion for $E\left\{\exp \left(-z \omega_{n}^{2}\right)\right\}$ with a formal inversion for $V_{n}()$. The former is claimed for all complex $z$ with real part $\mathcal{R}(z) \geqslant 0$. However, an inspection of the proof shows that it holds at best for real $z>0$. As a result of this, some terms are missing in the expansion for $V_{n}()$.

Finally, the expansion

$$
V_{n}(x)=V(x)+\sum_{j=1}^{r} \psi_{j}(x) n^{-j}+O\left(n^{-r-1}\right), \quad x \in \mathbf{R},
$$

was established by Götze (1979) for every fixed integer $r \geqslant 1$ and for some coefficient functions $\psi_{1}(), \psi_{2}(), \ldots$, where the remainder term is uniform in $x$. Whereas some basic techniques for estimating the remainder term are due to van Zwet (1977), Götze's basic innovation was the application of powerful Hilbert space ideas. In particular, $\Delta_{n}=O(1 / n)$ as $n \rightarrow \infty$, and this rate is unimprovable. For closely related theoretical results the reader is referred to Bentkus and Zitikis (1988), Zitikis (1988, 1989) and Bentkus et al. (1993).

Götze (1979) provided a general mathematical description of the coefficients, but the theoretical determination of $\psi_{2}(), \psi_{3}(), \ldots$ in terms of numerically computable functions appears to be hopeless. Therefore, we must resort to the one-term special case

$$
V_{n}(x)=V(x)+\frac{\psi_{1}(x)}{n}+O\left(\frac{1}{n^{2}}\right), \quad x \in \mathbf{R} .
$$

We shall claim that this should be sufficient for practical purposes. For this, with $\varphi()$ as in equation (1.2), Götze determines the Fourier-Stieltjes transform $\vartheta_{1}(t)=$ $\int_{0}^{\infty} \exp (\mathrm{it} x) \mathrm{d} \psi_{1}(x)$ as

$$
\vartheta_{1}(t)=\frac{1}{12} \varphi(t)-\frac{-2 \mathrm{i} t}{144} \varphi(t)-\frac{1}{36} \varphi^{3}(t)-\frac{1}{32} \varphi^{5}(t)-\frac{7 \cosh (-2 \mathrm{i} t)^{1 / 2}}{288} \varphi^{2}(t)
$$

for all $t \in \mathbf{R}$. For later reference, we note that if we extend Darling's function $a($ ) in equation (1.7) by considering $a(z), \mathcal{R}(z) \geqslant 0$, given by his formula, and put $\vartheta(t)=$ $a(-\mathrm{i} t)$ then $\vartheta() \neq \vartheta_{1}()$ on $\mathbf{R}$. Therefore, there is no theoretical basis for using an inverted form of Darling's (1960) heuristic approximation for the approximation of $V_{n}()$. 
As stated by Erdélyi et al. (1954), p. 246, equation (9), or Oberhettinger and Badii (1973), p. 259, equation (5.94), for all complex $z$ with $\mathcal{R}(z)>0$ and any constants $A>0$ and $v \in \mathbf{R}$,

$$
z^{v} \exp \left(-A z^{1 / 2}\right)=\int_{0}^{\infty} \exp (-z x)\left\{\frac{2^{-v-1 / 2}}{\pi^{1 / 2}} \frac{1}{x^{v+1}} \exp \left(\frac{-A^{2}}{8 x}\right) D_{2 v+1}\left(\frac{A}{2^{1 / 2} x^{1 / 2}}\right)\right\} \mathrm{d} x .
$$

Using this, Götze (1979) also gave a formula for $V(x)+\psi_{1}(x) n^{-1}$ in equation (1.8). However, his inversion is in error. For the leading term his formula gives half of what is in the third line of equation (1.3), and other terms also turned out to be similarly incorrect. Hence we must reinvert equation (1.9) here. Following Anderson and Darling's (1952) example 1, for all the terms in equation (1.9), with the simplification provided by the inversion formula above, we obtain

$$
\begin{aligned}
\psi_{1}(x)= & \frac{1}{12} V(x)-\frac{1}{9 \pi^{1 / 2} x^{3 / 4}} \sum_{k=0}^{\infty}(-1)^{k}\left(\begin{array}{c}
-\frac{3}{2} \\
k
\end{array}\right) \exp \left\{-\frac{(4 k+3)^{2}}{16 x}\right\} D_{1 / 2}\left(\frac{4 k+3}{2 x^{1 / 2}}\right) \\
& -\frac{7}{144 \pi^{1 / 2} x^{3 / 4}} \sum_{k=0}^{\infty}(-1)^{k}\left(\begin{array}{c}
-\frac{3}{2} \\
k
\end{array}\right) \exp \left\{-\frac{(4 k+1)^{2}}{16 x}\right\} D_{1 / 2}\left(\frac{4 k+1}{2 x^{1 / 2}}\right) \\
& -\frac{7}{144 \pi^{1 / 2} x^{3 / 4}} \sum_{k=0}^{\infty}(-1)^{k}\left(\begin{array}{c}
-\frac{3}{2} \\
k
\end{array}\right) \exp \left\{-\frac{(4 k+5)^{2}}{16 x}\right\} D_{1 / 2}\left(\frac{4 k+5}{2 x^{1 / 2}}\right) \\
& -\frac{1}{72 \pi^{1 / 2} x^{5 / 4}} \sum_{k=0}^{\infty}(-1)^{k}\left(\begin{array}{c}
-\frac{1}{2} \\
k
\end{array}\right) \exp \left\{-\frac{(4 k+1)^{2}}{16 x}\right\} D_{3 / 2}\left(\frac{4 k+1}{2 x^{1 / 2}}\right) \\
& -\frac{1}{4 \pi^{1 / 2} x^{5 / 4}} \sum_{k=0}^{\infty}(-1)^{k}\left(\begin{array}{c}
-\frac{5}{2} \\
k
\end{array}\right) \exp \left\{-\frac{(4 k+5)^{2}}{16 x}\right\} D_{3 / 2}\left(\frac{4 k+5}{2 x^{1 / 2}}\right)
\end{aligned}
$$

for all $x>0$. Although this formula lends itself to numerical computation in principle, most software packages do not contain built-in routines for the functions $D_{v}()$, whereas many such packages have routines for the Bessel functions $K_{v}()$ in equation (1.4). Using now the identities (1.5) and (1.6) and a standard property of the gamma function,

$$
\psi_{1}(x)=\frac{1}{12} V(x)+\frac{1}{\pi^{3 / 2}} \sum_{k=0}^{\infty} \frac{1}{k !}\left\{\frac{\Gamma\left(k+\frac{3}{2}\right) A_{k}(x)}{576 x^{3 / 2}}+\frac{B_{k}(x)}{2304 x^{5 / 2}}\right\}, \quad x>0,
$$

by lengthy but straightforward algebra, where, setting $G(y)=-\exp (-y)\left\{K_{1 / 4}(y)+\right.$ $\left.K_{3 / 4}(y)\right\}$ and $H(y)=\exp (-y)\left\{K_{5 / 4}(y)-3 K_{3 / 4}(y)-2 K_{1 / 4}(y)\right\}$ for all $y>0$, we have

$$
\begin{aligned}
A_{k}(x)= & 7(4 k+1)^{3 / 2} G\left\{\frac{(4 k+1)^{2}}{16 x}\right\}+16(4 k+3)^{3 / 2} G\left\{\frac{(4 k+3)^{2}}{16 x}\right\} \\
& +7(4 k+5)^{3 / 2} G\left\{\frac{(4 k+5)^{2}}{16 x}\right\}
\end{aligned}
$$


and

$$
B_{k}(x)=\Gamma\left(k+\frac{1}{2}\right)(4 k+1)^{5 / 2} H\left\{\frac{(4 k+1)^{2}}{16 x}\right\}+24 \Gamma\left(k+\frac{5}{2}\right)(4 k+5)^{5 / 2} H\left\{\frac{(4 k+5)^{2}}{16 x}\right\}
$$

\section{EXACT THEORY FOR $\omega_{n}^{2}$}

Introduce the simplex $S_{n}=\left\{\left(x_{1}, \ldots, x_{n}\right): 0 \leqslant x_{1} \leqslant \ldots \leqslant x_{n} \leqslant 1\right\}$, sitting in the unit cube of the $n$-dimensional space $\mathbf{R}^{n}$, and let $B_{n}\{c, \rho\}$ denote the $n$-dimensional closed ball with centre $c \in \mathbf{R}^{n}$ and radius $\rho \geqslant 0$. Let $\operatorname{vol}_{n}[]$ denote the $n$-dimensional Lebesgue measure. Since the joint density function of $U_{1, n}, \ldots, U_{1, n}$ is $n$ ! on $S_{n}$ and 0 otherwise, it follows from equation (1.1) that

$$
V_{n}(x)=n ! \int \ldots \int_{S_{n} \cap B_{n}\left\{c_{n}, \rho_{n}(x)\right\}} \mathrm{d} x_{1} \ldots \mathrm{d} x_{n}=n ! \operatorname{vol}_{n}\left[S_{n} \cap B_{n}\left\{c_{n}, \rho_{n}(x)\right\}\right]
$$

for every $x \geqslant 0$, where $c_{n}=(1 / 2 n, 3 / 2 n, \ldots,(2 n-1) / 2 n)$ and $\rho_{n}(x)=\{\max (0, x-$ $1 / 12 n)\}^{1 / 2}$. This was noticed by Marshall (1958), who has given exact formulae for $V_{1}()$ and $V_{2}\left(\right.$ ), and some indication for $V_{3}()$, where the analytical details are already quite formidable.

Little is known analytically about $V_{n}()$ for $n \geqslant 3$. Pearson and Stephens (1962) have given the first four moments for any $n$. Their and Tiku's (1965) approximations for the exact quantiles are discussed by Stephens and Maag (1968), whose lower tail probabilities are partially reproduced in equation (2.4) later. The approximate quantiles in Pearson and Stephens (1962), Stephens and Maag (1968) and Stephens (1970) are discussed by Knott (1974). Prokhorov (1968) proved that $\sup \left\{\left[1-V_{n}(x)\right]\right.$ : $n \geqslant 1\} \leqslant C \exp (-K x)$ for all $x>8 / \pi^{2} \approx 0.8106$, where $C=1+\exp (5 / 12) / \pi \sqrt{ } 2 \approx$ 1.3415 and $K=3 /(32 \exp 2) \approx 0.0126$. Since $C \exp (-K x) \leqslant 1$ only for $x \geqslant 23.3165$, this bound is non-trivial only for $n \geqslant 70$. But, it turns out, $1-V_{n}(x)$ is practically 0 for any $x \geqslant 1$, say, for $n$ so large.

The definitive paper on the distribution of $\omega_{n}^{2}$ for small sample sizes is by Knott (1974). His first step determines $\varphi_{n}(t)=E\left\{\exp \left(i t \omega_{n}^{2}\right)\right\}$ numerically for a large and sufficiently fine grid of $t$-values, based on equation (1.1) and numerical integration in the resulting recursion

$$
\begin{gathered}
I_{n, k+1}(t, x)=(k+1) \int_{0}^{x} \exp \left\{\mathrm{i} t\left(y-\frac{2 k+1}{2 n}\right)^{2}\right\} I_{n, k}(t, y) \mathrm{d} y, \\
t \in \mathbf{R}, \quad x \in[0,1], k=1, \ldots, n-1,
\end{gathered}
$$

giving $\varphi_{n}(t)=\exp (\mathrm{i} t / 12 n) I_{n, n}(t, 1)$, where

$$
I_{n, 1}(t, x)=\int_{0}^{x} \exp \left\{\mathrm{i} t\left(y-\frac{1}{2 n}\right)^{2}\right\} \mathrm{d} y
$$

and

$I_{n, k}(t, x)=k ! \int_{0}^{x} \int_{0}^{x_{k}} \ldots \int_{0}^{x_{2}} \exp \left\{\mathrm{i} t \sum_{j=1}^{k}\left(x_{j}-\frac{2 j-1}{2 n}\right)^{2}\right\} \mathrm{d} x_{1} \ldots \mathrm{d} x_{k}, \quad k=2, \ldots, n$,

and where we also correct some misprints in formulae (3.4)-(3.6) in Knott (1974). A 
more general form of this recursion, attributed to Knott, is also given by Durbin (1974), pages 27-28. The second step then uses the calculated complex values of $\varphi_{n}()$ in a numerical inversion formula to calculate a distribution function $V_{n}(\lambda ;)$, depending on a parameter $\lambda>0$, for which $\lim _{\lambda \rightarrow \infty}\left\{V_{n}(\lambda ; x)\right\}=V_{n}(x), x \in \mathbf{R}$. A suitable choice of $\lambda>0$ yields a numerical approximation of $V_{n}()$. Knott has accomplished this programme for $n=2, \ldots, 7$. With the choice of $\lambda=2$, his formula for the approximation of $V_{n}$ when $4 \leqslant n \leqslant 7$ is

$$
V_{n}(x) \approx V_{n}^{*}(x)=\frac{x}{2}+\frac{2}{\pi} \sum_{j=1}^{200} \frac{\sin (j x \pi / 2)}{j} \mathcal{R}\left\{\varphi_{n}\left(\frac{j \pi}{2}\right)\right\}, \quad 0<x<2,
$$

where $V_{n}(x) \leqslant V_{n}^{*}(x) \leqslant V_{n}(x)+1-V_{n}(2)$ for all $0<x<2$. The functions $V_{1}()$ and $V_{2}($ ) are also available from Marshall (1958) exactly, allowing for the misprint in $V_{2}$ ( ) spotted by Marshall and reported in a footnote in Pearson and Stephens (1962), p. 400 .

Assuming that Darling's approximation above equation (1.7) is valid, and in fact that it holds for all complex $z$ with $\mathcal{R}(z) \geqslant 0, \operatorname{Knott}(1974)$ supposed that for $n \geqslant 8$, say, $\varphi(t)+\vartheta(t) n^{-1}$ is a good approximation of $\varphi_{n}(t), t \in \mathbf{R}$, where $\vartheta(t)=a(-\mathrm{i} t)$. So he also inverted $\varphi()+\vartheta() n^{-1}$, using the second step above, to obtain an approximation to $V_{n}()$ for $n \geqslant 8$. As pointed out following equation (1.9), the correct function to do this is not $\varphi()+\vartheta() n^{-1}$ but $\varphi()+\vartheta_{1}() n^{-1}$. This means that all Knott's (1974) considerations in his section 4, including his Table 1 and its discussion, are in error, as are all the percentage points given for $n=8,9,10,20,50$, 200 and 1000 in his Table 2 in section 5.

Returning to exact distributions, set $\Lambda_{k}(c, \rho)=\operatorname{vol}_{k}\left[S_{k} \cap B_{k}\{c, \rho\}\right]$ for some $c \in \mathbf{R}^{k}$ and $\rho \geqslant 0, k=1,2, \ldots$, so that $V_{n}(x)=n ! \Lambda_{n}\left\{c_{n}, \rho_{n}(x)\right\}, x \geqslant 0$, by equation (2.1). Let $c_{k}=c_{n-1, k}$ be the orthogonal projection of $c_{n}$ onto the $(n-1)$-dimensional hyperplane in which the $k$ th face $S_{n-1, k}$ of $S_{n}$ lies, let $\alpha_{k}=\alpha_{n-1, k}$ be the $n$-dimensional distance between $c_{n}$ and $c_{k}=c_{n-1, k}$, and put $\Lambda_{n-1, k}\left(c_{k}, \rho\right)=\operatorname{vol}_{n-1}\left[S_{n-1, k} \cap B_{n-1}\left\{c_{k}, \rho\right\}\right]$ for any $\rho \geqslant 0, k=1, \ldots, n+1$. Our method of computing $V_{n}()$ is based on the following recursive formula given in Csörgő and Stachó (1980): for every integer $n \geqslant 1$ and $x \geqslant 0$,

$$
\Lambda_{n}\left\{c_{n}, \rho_{n}(x)\right\}=\rho_{n}^{n}(x)\left(\frac{\pi^{n / 2}}{\Gamma(n / 2+1)}-\sum_{k=1}^{n+1} \alpha_{k} \int_{0}^{\rho_{n}(x)} \frac{\Lambda_{n-1, k}\left[c_{k},\left\{\left(y^{2}-\alpha_{k}^{2}\right)^{+}\right\}^{1 / 2}\right]}{y^{n+1}} \mathrm{~d} y\right)
$$

The same applies to all of $\Lambda_{n-1, k}\left\{c_{k}, \rho_{k}(y)\right\}$ for the radii $\rho_{k}(y)=$ $\left\{\max \left(0, y^{2}-\alpha_{k}^{2}\right)\right\}^{1 / 2}, 0 \leqslant y \leqslant \rho_{n}(x), k=1, \ldots, n+1$, reducing the dimension to $n-2$, and so on.

Of course, we recognize that

$$
\frac{\rho_{n}^{n}(x) \pi^{n / 2}}{\Gamma(n / 2+1)}=\operatorname{vol}_{n}\left[B_{n}\left\{c_{n}, \rho_{n}(x)\right\}\right]
$$

and all the integrals in equation (2.3) are 0 if $x$ is so small that $\rho_{n}(x) \leqslant \alpha_{k}=\alpha_{n-1, k}$, 
$k=1, \ldots, n+1$. It is easy to see that, in a suitable listing of the faces, $\alpha_{n-1,1}=$ $\alpha_{n-1,2}=1 / 2 n$ and $\alpha_{n-1,3}=\ldots=\alpha_{n-1, n+1}=1 / n \sqrt{ } 2$. Thus, solving $\rho_{n}(x)=1 / 2 n$, it follows that

$$
V_{n}(x)=\frac{n ! \pi^{n / 2}}{\Gamma(n / 2+1)}\left(x-\frac{1}{12 n}\right)^{n / 2}, \quad \frac{1}{12 n} \leqslant x \leqslant \frac{n+3}{12 n^{2}} .
$$

Finally, let $d(n)=\lceil n / 2\rceil-1$, where $\lceil n / 2\rceil$ is the smallest integer not smaller than $n / 2$. The corollary in Csörgö and Stachó (1980), or its proof as applied to formula (2.3), implies that function $V_{n}()$ is exactly $d(n)$ times continuously differentiable on $\mathbf{R}$. That the result is unimprovable follows from equation (2.4). Generalizations can be found in Bentkus and Zitikis (1988) and Bentkus et al. (1993). (The original result is incorrectly stated in Csörgö and Stachó (1980), claiming that $V_{n}()$ is $\lfloor n / 2\rfloor$ times continuously differentiable, where $\lfloor n / 2\rfloor$ is the usual integer part of $n / 2$.) Note that $d(1)=d(2)=0$, so $V_{1}$ and $V_{2}$ are only continuous, $d(3)=d(4)=1, d(5)=d(6)=2$, $d(7)=d(8)=3$, and so on.

\section{RESULTS AND DISCUSSION FOR $\omega_{n}^{2}$}

The linking approximation (1.8) requires only the direct computation of the functions $V(x)$ and $\psi_{1}(x)$. Working with the second formula in equation (1.3) and with equation (1.10), large precision numerical stability sets in for any $x>0$, using just a few terms of the infinite series. As a by-product, we found all five digits in Martynov's (1978) Table 1 to be precise for all 250 values $V(0.000)=0.00000, V(0.005)=$ $0.00000, V(0.010)=0.00001, \ldots, V(0.995)=0.99747, V(1.00)=0.99754, V(1.02)=$ $0.99779, \ldots, V(1.98)=0.99999$ given there. In contrast, the integrations in the geometric recursion (2.3) for the exact distribution function $V_{n}(x), 1 / 12 n \leqslant x \leqslant n / 3$, must be done numerically and each evaluation of the integrand then requires a further numerical integration and so on. As discussed earlier, the integrands are not smooth and we cannot determine in advance the points at which discontinuous derivatives occur, so we can do no better than a crude trapezoidal rule for the numerical integrations. The speed of the computation is heavily dependent on $x$. As $x$ becomes larger the ball intersects the simplex in an increasingly complicated way and the recursive numerical integrations become more extensive.

The lower numbers in the double entries in Table 1 for $n=2, \ldots, 8$ are obtained by the exact geometric recursion described above, except for those printed in italics or marked by an asterisk. For $n=2$ we used Marshall's (1958) explicit formula, with the correction in Pearson and Stephens (1962) referred to earlier, and for certain small values of $p$ we used equation (2.4) directly. Whenever the numbers from the geometric computation are present in Table 1 , they agree with the exact results in Table 2 of Knott (1974) for $2 \leqslant n \leqslant 7$ and $0.01 \leqslant p \leqslant 0.99$ within three units in the fifth decimal place, except in a few cases of small $x$, where it was easy to see that Knott's numbers are incorrect since only the volume of the ball is needed. The computational cost to achieve this accuracy became too great for the missing values, so the italic numbers appearing in Table 1 are taken from Table 2 of Knott (1974), which he obtained by using his formula (2.2) above. However, the accuracy of 
Knott's computation is independent of $p$ or $x$, and hence it can be safely supposed that none of these italic numbers are in error, either, by more than three units in the fifth decimal place. Whenever we decided to report one of Knott's numbers, we approached sufficiently close to it until it became clear that finer grids of the numerical integrations and weeks of additional computer time would give that number.

To check on this point further and to produce some more 'exact' values for comparison for $n=8,9,10$, as well as a new column for the very extreme case of $p=0.999$ which is not covered by Knott (1974), we have also conducted a rather large scale, and much cheaper, simulation study. For each $n=2, \ldots, 10$ as sample sizes, 99999 pseudo-independent replicas of $\omega_{n}^{2}$ were simulated and quantiles were estimated from the sorted statistics. This was repeated 4000 times, over which these quantiles were averaged to produce the final estimated quantiles. For $p=0.999$ and $n=8,9,10$, these appear in Table 1 with four decimal places and marked by asterisks. The standard error was no more than 0.00008 for $p<0.999$ and no more than 0.00024 for $p=0.999$. The simulation results confirm all the exact numbers up to $n=8$ in Table 1 to their fourth decimal.

For a given $n$ and $0<p<1$, the approximate $p$-quantile of $\omega_{n}^{2}$ provided by the one-term linking approximation (1.8) is the $x=x(n, p)$ for which $n V(x)+\psi_{1}(x)=$ $n p$. There is a unique root $x_{0}$ so that $\psi_{1}\left(x_{0}\right)=0, \psi_{1}(x)<0$ if $x<x_{0}$ and $\psi_{1}(x)>0$ if $x>x_{0}$. We obtain $x_{0}=0.26702$ and $p_{0}=V\left(x_{0}\right)=0.83208$. So the $p_{0}$-quantiles given by the approximation and by the asymptotic distribution are the same. If $p<p_{0}$, then the approximation corrects the asymptotic $p$-quantile by producing a greater value, and if $p>p_{0}$ then the corrected value is smaller than the asymptotic $p$ quantile. The upper numbers in the double entries in Table 1 for $n=2, \ldots, 10$ and the single entries for $n \geqslant 20$ are those obtained by the one-term linking approximation.

As can be seen from Table 1, the linking approximation agrees closely with the exact values even for very small $n$. Fig. 1 shows the accuracy of this approximation for $n=2,3,4,5$. We plot $n\left\{V_{n}(x)-V(x)\right\}$ on the vertical axis to examine how closely this is approximated by $\psi_{1}(x)$. For $n=7$, the largest deviation between the exact and the corrected asymptotic $p$-quantiles is 0.00016 for $p<0.9$, and it is 0.00237 for $0.9 \leqslant p \leqslant 0.99$. For large $p$ the small error will lead to slightly conservative $p$-values.

\section{WATSON'S STATISTIC $u_{n}^{2}$}

Watson's (1961) modification of the Cramér-von Mises statistic $\omega_{n}^{2}$ is

$$
u_{n}^{2}=n \int_{-\infty}^{\infty}\left[F_{n}(x)-F(x)-\int_{-\infty}^{\infty}\left\{F_{n}(y)-F(y)\right\} \mathrm{d} F(y)\right]^{2} \mathrm{~d} F(x),
$$

for which, with the continuity assumption on $F$ and the notation in Section 1,

$$
\frac{1}{12 n} \leqslant u_{n}^{2}=\frac{1}{12 n}+\sum_{k=1}^{n}\left(U_{k, n}-\frac{2 k-1}{2 n}\right)^{2}-n\left(\frac{1}{n} \sum_{k=1}^{n} U_{k}-\frac{1}{2}\right)^{2} \leqslant \frac{n}{12}, \quad n=1,2, \ldots
$$




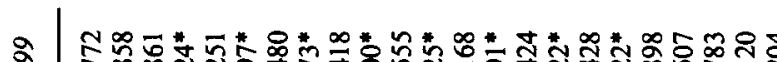

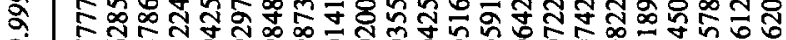

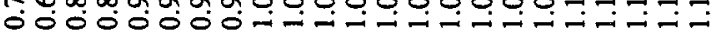

-

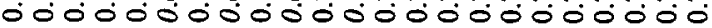

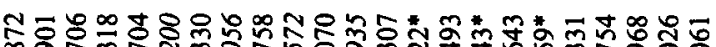

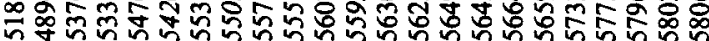

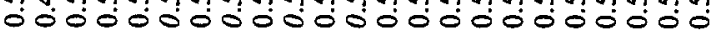

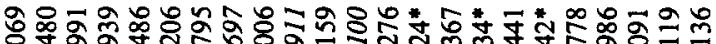
00000000000000000000000

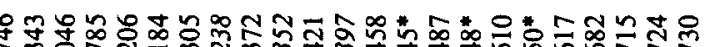

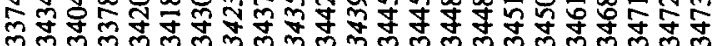

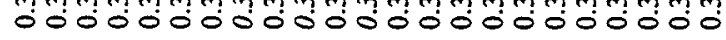

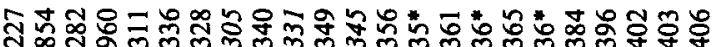

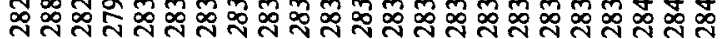
00000000000000000000000

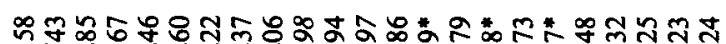

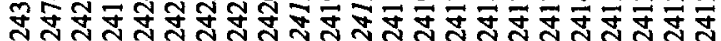

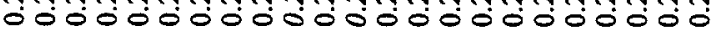

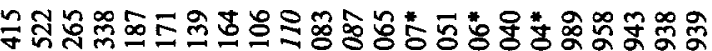

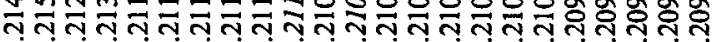

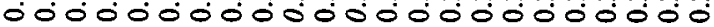

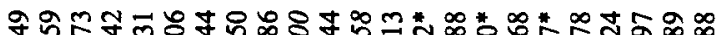

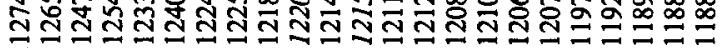

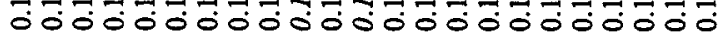

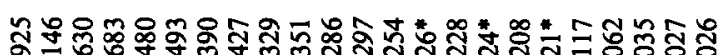

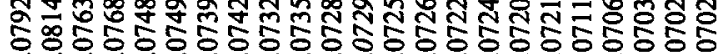
o-000000000000000000000

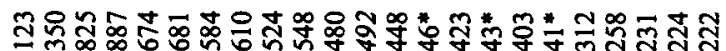

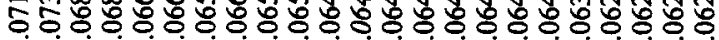

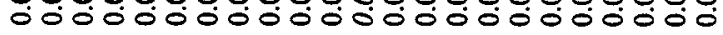

유

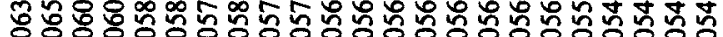

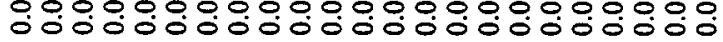

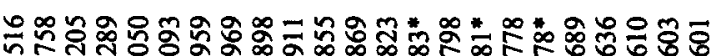

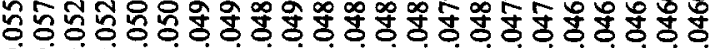

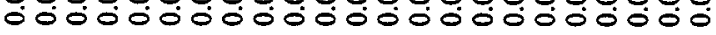

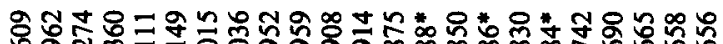

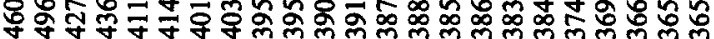

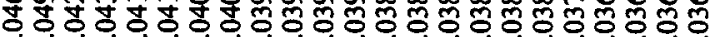

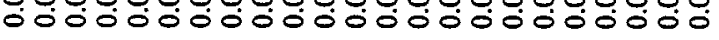

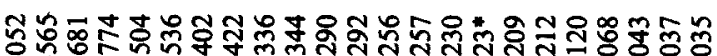

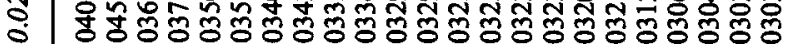

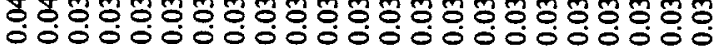

○

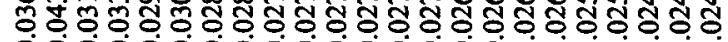
0.00000000000000000000000 


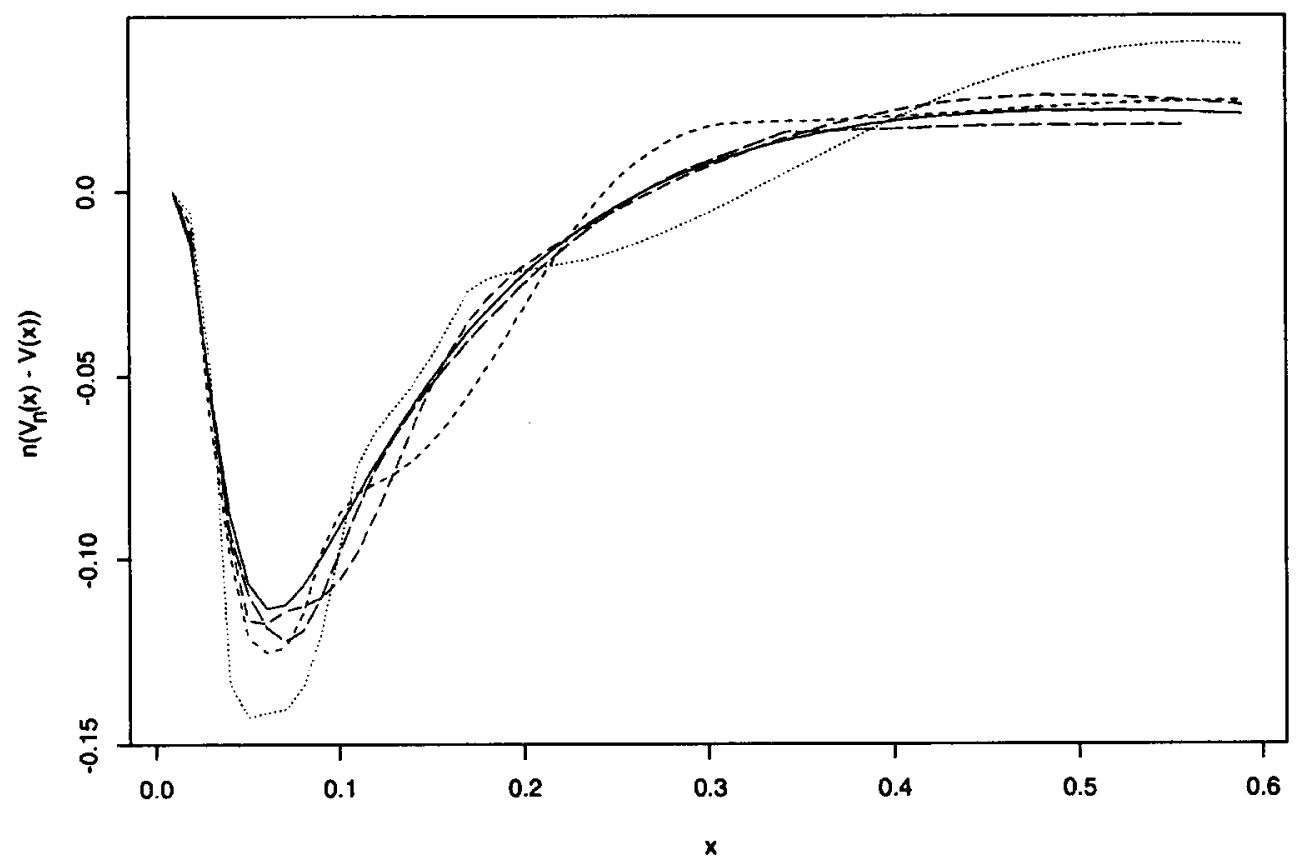

Fig. 1. Comparison of the linking approximation to the exact distribution for $\omega_{n}^{2}: \cdots \cdots \cdots, n=2 ; \ldots \ldots$, $n=3 ;---, n=4 ;-, n=5 ; \stackrel{-}{\longrightarrow}, \psi_{1}(x)$

The 'correction for the mean' makes $u_{n}^{2}$ rotationally invariant in that, when it is adapted for testing goodness of fit on the unit circumference of a circle, it is independent of the origin of polar co-ordinates, the starting point for cumulating distributions. Setting $W_{n}(x)=P\left\{u_{n}^{2} \leqslant x\right\}$ and $W(x)=P\left\{u^{2} \leqslant x\right\}$, where

$$
u^{2}=\int_{0}^{1} B^{2}(s) \mathrm{d} s-\left\{\int_{0}^{1} B(s) \mathrm{d} s\right\}^{2}
$$

for a Brownian bridge $B($ ) and $x \in \mathbf{R}$, Watson (1961) proved that

$$
\lim _{n \rightarrow \infty}\left\{W_{n}(x)\right\}=W(x)=1+2 \sum_{k=1}^{\infty}(-1)^{k} \exp \left(-2 k^{2} \pi^{2} x\right), \quad x>0
$$

A table for $W()$ is given in Martynov (1978), p. 70.

Results on the exact distribution of $u_{n}^{2}$ for a finite sample size $n$ have been obtained by Pearson and Stephens (1962), Stephens $(1963,1964,1970)$ and Tiku (1965). These approximations are either based on moments or are empirical. Of more importance for our purposes is that, by an ingenious geometric insight, Stephens $(1963,1964)$ could give exact formulae for $W_{2}(), W_{3}()$ and $W_{4}()$. In contrast, again as a special case of a complete asymptotic expansion, Götze (1979) established that 


$$
W_{n}(x)=W(x)+\frac{\psi(x)}{n}+O\left(\frac{1}{n^{2}}\right), \quad x \in \mathbf{R},
$$

where the remainder term is uniform in $x$ and, with $w()=W^{\prime}()$ denoting the density function of $W()$ and $w^{\prime}()$ standing for the derivative of $w()$,

$$
\begin{aligned}
\psi(x) & =\left(\frac{1}{144}-\frac{5}{12} x\right) w(x)-\frac{1}{6} x^{2} w^{\prime}(x) \\
& =\frac{\pi^{2}}{3} \sum_{k=1}^{\infty}(-1)^{k}\left(5 x-4 k^{2} \pi^{2} x^{2}-\frac{1}{12}\right) k^{2} \exp \left(-2 k^{2} \pi^{2} x\right) .
\end{aligned}
$$

The coefficient functions of $n^{-2}, n^{-3}, \ldots$ in Götze's full expansion are not known explicitly, but it will be suggested again that the one-term expansion in equation (4.1) is all that is needed. Limited information on this has already been given by Götze (1979) himself.

The lower numbers in the double entries in Table 2 for $n=2,3,4$ are obtained from programming Stephens's $(1963,1964)$ exact formulae. Here we use the occasion to point out a misprint in Stephens's (1964) formula on p. 396 for $n=4$ on the interval $[7 / 48,1 / 3]$, where $1 / 48$ in the argument of the second inverse tangent should be $7 / 48$. The other exact five-decimal lower numbers for $n=5, \ldots, 9$ have been obtained by using Stephens's (1964) exact lower tail formulae on p. 397; these have also been recomputed because the corresponding numbers in Table 2 of Stephens (1964) contain only four decimal places. The accompanying simulation study went as for $\omega_{n}^{2}$, but here the procedure was repeated only 1000 times instead of 4000 because the simulation for $u_{n}^{2}$ was less variable. The corresponding estimated quantiles appear as the lower numbers in Table 2 for $n=5, \ldots, 10$, with four decimal places and marked by asterisks. For $n=2,3,4$, the largest difference between the recorded exact numbers and their simulated counterparts was 0.00009 for $p \leqslant 0.99$ and 0.00016 for $p=0.999$. In the whole simulation, the standard error was no more than 0.00004 for $p<0.999$ and no more than 0.00016 for $p=0.999$. None of the simulated numbers are thought to be in error by more than one unit in the fourth decimal place.

Working with equation (4.1), we found all five digits in Martynov's (1978) Table 11 to be precise for all 200 values $W(0.000)=0.00000, W(0.002)=0.00000, \ldots$, $W(0.298)=0.99442, W(0.300)=0.99464, W(0.305)=0.99514, \ldots, W(0.545)=$ 0.99996 given there. Again, for a given $n$ and $p \in(0,1)$, the approximate $p$-quantile of $u_{n}^{2}$ provided by the linking approximation (4.1) is the $y=y(n, p)$ for which $n W(y)+\psi(y)=n p$. The upper numbers in the double entries in Table 2 for $n=2, \ldots, 10$ and the single entries for $n \geqslant 20$ result from this. As for $\omega_{n}^{2}$, there is a unique root $y_{0}$ so that $\psi\left(y_{0}\right)=0, \psi(y)<0$ if $y<y_{0}$ and $\psi(y)>0$ if $y>y_{0}$, where $y_{0}=0.10938$ and $W\left(y_{0}\right)=0.76949$.

The approximation of the exact percentage points is close even for $n=4$. Fig. 2 plots $\psi(x)$ and $n\left\{W_{n}(x)-W(x)\right\}$ for $n=2,3,4,5$ to see this. For $n=4$, the largest deviation between the exact and the corrected asymptotic $p$-quantiles is 0.00074 for $p<0.9$, and it is 0.00446 for $0.9 \leqslant p \leqslant 0.99$. The corresponding deviations for $n=7$, using the original simulated numbers before rounding off, are 0.00012 and 0.00283 . The accuracy is better for $n=4$ and is about the same for $n=7$ in 
[No. 1,

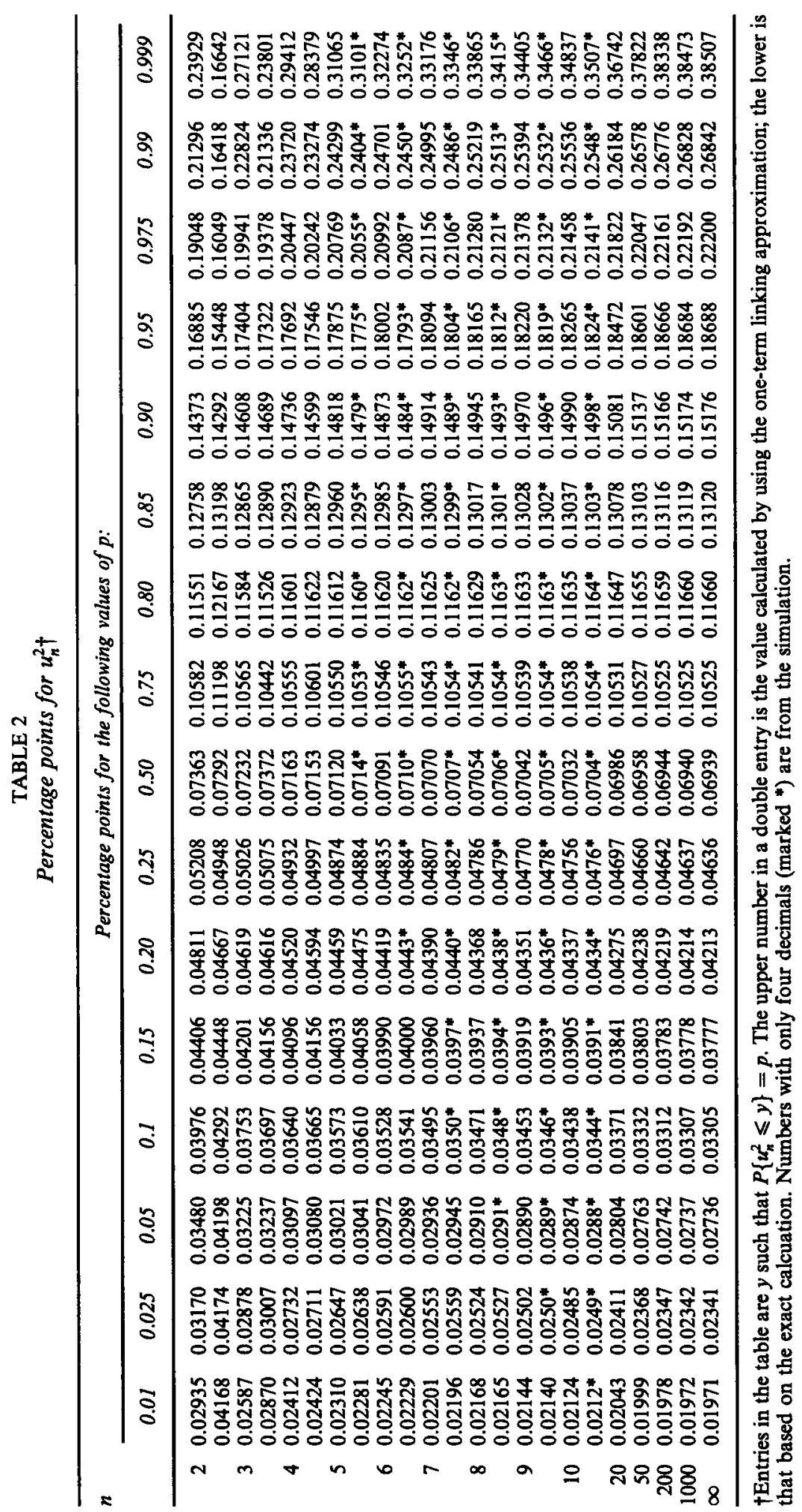




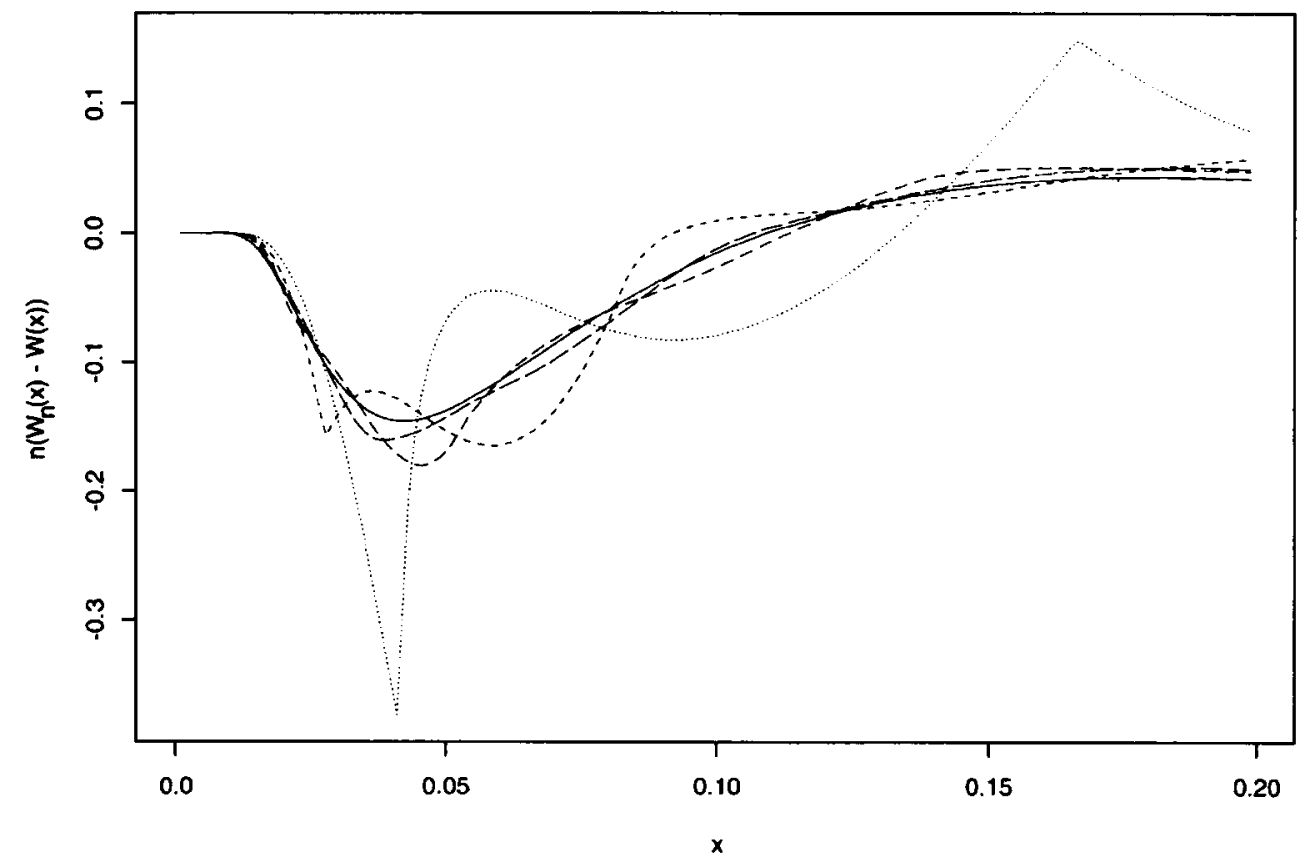

Fig. 2. Comparison of the linking approximation to the exact distribution for $u_{n}^{2}: \cdots \cdots \cdots, n=2 ;-\ldots,-$, $n=3 ;-\cdots, n=4 ;-\cdots, n=5 ;-, \psi(x)$

comparison with the corresponding accuracy for $\omega_{n}^{2}$. Also, for large $p$ the small error will again lead to slightly conservative $p$-values.

Since the one-term linking approximations may be computed in a few seconds on modern equipment, accurate $p$-values for the Cramér-von Mises and Watson statistics may be obtained quickly for the sample sizes that would be considered in practice. The programs computing $V(x)+\psi_{1}(x) n^{-1}$ and $W(x)+\psi(x) n^{-1}$ for any given $x>0$ and $x=x(n, p)$ and $y=y(n, p)$ solving $n V(x)+\psi_{1}(x)=n p$ and $n W(y)+\psi(y)=n p$ for any given $0<p<1$ and $n$ will be made available publicly.

\section{ACKNOWLEDGEMENT}

This research was supported in part by National Science Foundation grant DMS9208067.

\section{REFERENCES}

Abramowitz, M. and Stegun, I. A. (1973) Handbook of Mathematical Functions. New York: Dover Publications.

Anderson, T. W. and Darling, D. A. (1952) Asymptotic theory of certain "goodness of fit" criteria based on stochastic processes. Ann. Math. Statist., 23, 193-212.

Bentkus, V., Götze, F. and Zitikis, R. (1993) Asymptotic expansions in the integral and local limit theorems in Banach spaces with applications to $\omega$ statistics. J. Theor. Probab., 6, 727-780.

Bentkus, V. and Zitikis, R. (1988) Remark on the Cramér-von Mises-Smirnov criterion. Lith. Math. J., 28, 8-13.

Cramér, H. (1928) On the composition of elementary errors: II, Statistical applications. Skand. Akt., 11, 141-180. 
Csörg6, S. (1975) Asymptotic expansion for the Laplace transform of the von Mises $\omega^{2}$ criterion. Theory Probab. Applic., 20, 158-161.

(1976) On an asymptotic expansion for the von Mises $\omega^{2}$ statistic. Acta Sci. Math. (Szeged), 38, 45-67.

Csörgö, S. and Stachó, L. (1980) A step toward an asymptotic expansion for the Cramér-von Mises statistic. In Colloq. Math. Soc. J. Bolyai, 21, 53-65.

Darling, D. A. (1960) On the theorems of Kolmogorov-Smirnov. Theory Probab. Applic., 5, 356-361.

Durbin, J. (1973) Distribution theory for tests based on the sample distribution function. Regl Conf. Ser. Appl. Math., 9.

Erdélyi, A., Magnus, W., Oberhettinger, F. and Tricomi, F. G. (1954) Tables of Integral Transforms, vol. 1. New York: McGraw-Hill.

Götze, F. (1979) Asymptotic expansions for bivariate von Mises functionals. Z. Wahrsch. Ver. Geb., 50, 333-355.

Knott, M. (1974) The distribution of the Cramér-von Mises statistic for small sample sizes. J. R. Statist. Soc. B, 36, 430-438.

Marshall, A. W. (1958) The small sample distribution of $n \omega_{n}^{2}$. Ann. Math. Statist., 29, 307-309.

Martynov, G. V. (1978) Omega-square Criteria (in Russian). Moscow: Nauka.

von Mises, R. (1931) Wahrscheinlichkeitsrechnung und Ihre Anwendung in der Statistik und Theoretischen Physik. Leipzig: Deuticke.

(1947) Differentiable statistical functions. Ann. Math. Statist., 18, 309-348.

Oberhettinger, F. and Badii, L. (1973) Tables of Laplace Transforms. New York: Springer.

Pearson, E. S. and Stephens, M. A. (1962) The goodness-of-fit tests based on $W_{n}^{2}$ and $U_{n}^{2}$. Biometrika, 49, 397-402.

Prokhorov, Yu. V. (1968) An extension of S. N. Bernstein's inequalities to the multidimensional case. Theory Probab. Applic., 13, 260-267.

Smirnov, N. V. (1936) Sur la distribution de $\omega^{2}$ (critérium de M. R. v. Mises). C. R. Acad. Sci. Paris, 202, 449-452.

(1937) On the distribution of Mises' $\omega^{2}$-criterion (in Russian). Mat. Sb. (Nov. Ser.), 2, 973-993. Stephens, M. A. (1963) The distribution of the goodness-of-fit statistic $U_{n}^{2}$ : I. Biometrika, 50, 303-313. (1964) The distribution of the goodness-of-fit statistic $U_{n}^{2}$ : II. Biometrika, 51, 393-397.

(1970) Use of the Kolmogorov-Smirnov, Cramér-von Mises and related statistics without extensive tables. J. R. Statist. Soc. B, 32, 115-122.

Stephens, M. A. and Maag, U. R. (1968) Further percentage points for $W_{n}^{2}$. Biometrika, 55, 428-430.

Tiku, M. L. (1965) Chi-square approximations for the distributions of goodness-of-fit statistics $W_{n}^{2}$ and $U_{n}^{2}$. Biometrika, 52, 630-633.

Watson, G. N. (1966) $A$ Treatise on the Theory of Bessel Functions, 2nd edn. Cambridge: Cambridge University Press.

Watson, G. S. (1961) Goodness-of-fit tests on a circle. Biometrika, 48, 109-114.

Zitikis, R. (1988) Asymptotic expansions in the local limit theorem for $\omega_{n}^{2}$ statistics (in Russian). Litovsk. Mat. Sb., 28, 461-474.

(1989) Asymptotic expansions for the derivatives of the Anderson-Darling statistic's distribution function (in Russian). Litovsk. Mat. Sb., 29, 33-53.

van Zwet, W. R. (1977) Asymptotic expansions for the distribution functions of linear combinations of order statistics. In Statistical Decision Theory and Related Topics II (eds S. S. Gupta and D. S. Moore), pp. 421-437. New York: Academic Press. 